\title{
Peter Eichhorn
}

\section{La gestión empresarial aplicada a los establecimientos estatales $(*)$}

SUMARIO: I. LA ACTIVIDAD ESTATAL. II. PRESUPUESTACION NETA. III. EL PRINCIPIO DE EQUTVALENCIA. IV. COMPARACION DE LA EFICACIA. V. OBJETTVOS DE LA PRESTACION. VI. PRINCIPIO DE CONGRUENCIA. VII. INCENTIVOS DE PRODUCCION. VIII. DETERMINACION DEL RESULTADO. IX. SUBVENCIONES GLOBALES.

\section{LA ACTIVIDAD ESTATAL}

Nos encontramos aquí ante una materia muy interesante: mientras que en la práctica disputan los funcionarios ministeriales con el personal de los establecimientos estatales sobre la forma y el método de una economía funcional, a los científicos interesa la controvertida cuestión de dilucidar si y hasta qué punto han de ser dirigidos determinados establecimientos estatales de forma cameralística o más bien comercial.

Una cuestión que históricamente no aparecía formulada en términos contradictorios. En los siglos XVII y XVIII la tarea de los cameralistas de los príncipes absolutos consistía en dirigir las propiedades, canteras, salinas, minas, manufacturas de porcelana, telares, papel, armas y municiones, etc., de tal manera que las necesidades estatales con-

(*) Articulo publicado en la revista Schriften zur öffentlichen Verwaltung und öffentlichen Wirtschaft: Studentenwerke und Universitätskliniken im Konflikt mit dem Landeshaushaltsrecht, Peter EICHHORN (editor), tomo 89, Baden-Baden, 1986, pp. 21-31.

El INAP y la redacción de DOCUMENTACION ADMINISTRATIVA desean dejar constancia de su agradecimiento al autor por la autorización concedida para la traducción al castellano y publicación del presente trabajo. 
secuentes al costoso mantenimiento de la corte pudiesen ser satisfechas directamente, asignándose las ganancias obtenidas con la venta de los productos producidos a los presupuestos destinados a la creación de un funcionariado profesional y del Ejército. Los cameralistas pensaban y actuaban, por tanto, en términos comerciales al servicio de los monarcas.

Aunque ya pertenece a la historia la época de l'etat c'est moi, se ha conservado la participación del Estado en los sectores económicos. Las razones existentes para ello no son del todo pacíficas y pueden deberse al desarrollo histórico concreto o ser explicables desde la perspectiva de las exigencias actuales. A cuyo efecto basta pensar en las empresas y participaciones en la economía de crédito y abastecimiento, en la construcción de viviendas y urbanismo, en el desarrollo agrario y el fomento de la economía, en la política de abastecimiento y transportes, en la investigación, en la tecnología y en el sector de servicios (como, por ejemplo, empresas estatales de exposiciones, congresos, ferias y publicidad, casas de juego, loterías, hostelería, hoteles, empresas de abastecimiento, consulting, información, editorial y de control financiero).

Entre los defensores de la titularidad estatal de las empresas y, sobre todo, entre los que apoyan la privatización de estas empresas se ha llegado a cierta concordancia sobre la necesidad y oportunidad de la gestión comercial. Pero si bien existe un acuerdo hasta aquí, no sucede, en cambio, lo mismo en relación a los llamados establecimientos estatales, sobre los cuales discrepan los criterios. Se trata de instituciones que de ordinario más que realizar las tareas estatales de forma administrativa lo hacen a cambio de remuneración. Las discrepancias empiezan ya por el hecho de que determinadas unidades organizativas, $y$ sobre todo en los sectores de sanidad, social y cultural, suelen ser denominados por unos como establecimientos estatales o del Land, mientras que otros, por el contrario, consideran que el término «empresa» sería más adecuado a sus actividades.

$Y$ no nos encontramos ante una polémica simplemente verbal, sino que en ella se debaten cuestiones fundamentales sobre cómo han de ser realizadas las funciones públicas. Nuestra Constitución y el orden económico existente nos brindan en principio las siguientes posibilidades: las funciones públicas pueden ser realizadas por instituciones públicas o se encomiendan a economías domésticas y entes privados (como, por ejemplo, peritos, ingenieros con examen estatal y empresas concesionarias). En la primera opción deberá determinarse asimismo si esas tareas tienen que ser llevadas a cabo con medios burocráticos o por empresas públicas (estatales o locales). Por supuesto, también la segunda opción nos ofrece algunas variaciones (que aquí no serán estudiadas con el detalle que requerirían). Pero el verdadero problema 
que se nos presenta se debe a que los establecimientos estatales se encuentran en una zona limítrofe entre la Administración y la economía, es decir, entre el Estado y el mercado. Se trata de una situación ambigua, dado que las funciones estatales, por un lado, se dirigen por el gobierno del Land y la Administración ministerial; pero, por otro, han de gestionarse con instrumentos empresariales. Los miembros de la Administración titular refuerzan la supervisión estatal mientras que los dirigentes de los establecimientos estatales subrayan más las funciones de management en la dirección de la empresa.

\section{PRESUPUESTACION NETA}

En favor de la naturaleza empresarial de un establecimiento industrial hablan las siguientes circunstancias y casos: en primer término, cuando se dispone de un capital propio, que pueda reproducirse, al menos parcialmente, en el mercado (lo que corresponde, en última instancia, a cualquier corporación territorial) y, en segundo lugar, cuando también se dispone de un marco autorresponsable de actuación y decisión. Lo primero se reflejará en una posición económico-financiera y contable especial respecto del presupuesto del Ente matriz, y, a su vez, esta presupuestación neta se verá acompañada en muchos aspectos de una cierta independencia organizativa respecto de la Administración titular.

En un sistema de presupuestación neta no aparecen en el presupuesto los ingresos ni los gastos de la empresa (neta). La empresa planifica generalmente con categorías mercantiles, es decir, con rentas e inversiones, ingresos y gastos, así como con las existencias y cambios del capital y de las deudas. En el plan presupuestario del Ente matriz sólo se recogen las figuras de subvenciones y transferencias. Se trata, por tanto, de una excepción al principio de integridad y unidad del presupuesto recogido en el $\$ 8$ del Haushaltsgrundgesetz (HGrG) (Ley Fundamental de Presupuestos) de 19 de agosto de 1969 (BGB1. I S. 1273).

El $\$ 26$ de la Bundeshaushaltsordnung (BHO) (Reglamento Presupuestario Federal) de 19 de agosto de 1969 (BGBl. I S. 1284) y, con el mismo contenido textual las Landeshaushaltsordnungen (LHO) (Reglamento Presupuestario del Land), prevé en su primer apartado, a propósito del alcance de la presupuestación neta, que los entes estatales y, en su caso, locales aprobarán un plan económico que deberá incluir, por un lado, un plan de resultados $y$, por otro, uno financiero o de capital. Conforme a esta práctica, consolidada ya desde hace decenios en las empresas propias municipales, es frecuente que los patrimonios especiales y las corporaciones, instituciones y fundaciones de Derecho 
público formulen igualmente un plan económico que, además de los gastos que ya se han indicado, comprenda una relación de los puestos de trabajo de los empleados, trabajadores y funcionarios (aunque, por lo que a estos últimos se refiere, tenga un contenido meramente informativo, puesto que han de aparecer en la relación de puestos de trabajo del Ente matriz).

Los establecimientos estatales que según el $\$ 26$, apartado $1 .^{\circ}$, LHO actúen con forma de empresa jurídicamente dependiente son, por tanto, una parte de la Administración del Land y su gestión, en lo que a sus puestos de trabajo se refiere, está sometida principalmente a las regulaciones presupuestarias (por ejemplo, prohibiciones de aumentos y reducciones de plantilla). Los establecimientos estatales que se rijan, en cambio, como patrimonio especial por el $\$ 26$, apartado $2 .^{\circ}$, LHO o como corporaciones, instituciones y fundaciones de Derecho público jurídicamente independientes son una parte integrante del Patrimonio del Land y disponen de autonomía suficiente para la gestión de sus medios financieros y personales disponibles.

\section{EL PRINCIPIO DE EQUIVALENCIA}

Para que sea posible una gestión económica es preciso que se den las formas adecuadas de los establecimientos estatales y, sobre ello, la concurrencia de los demás presupuestos económicos necesarios a tal fin. Ya hemos mencionado la reproducción del capital propio, adecuada al mercado, al menos parcialmente. Ello quiere decir, en otras palabras, que las prestaciones se entregan a cambio de unas contrasprestaciones. De esta forma las empresas se financian con tales equivalencias, mientras que las Administraciones lo hacen con prestaciones públicas que carecen de la correspondiente contraprestación. Pero junto a estas figuras aparecen formas mixtas. Así, algunos establecimientos - como los de ayuda al estudiante, clínicas universitarias, hospitales psiquiátricos de los Lander, sanatorios, puertos interiores, establecimientos experimentales - realizan sus prestaciones en parte con ánimo de lucro cubriendo parcial o completamente los costes, en parte de manera gratuita, recibiendo, en cambio, por ello pagos globales o compensatorios, devoluciones, subvenciones, asignaciones y otro tipo de pagos. La ventaja fundamental del principio de equivalencia es la igualación entre oferta y demanda que se alcanza por los mecanismos del mercado en contraposición al principio impositivo o regaliano. Al mismo tiempo, la empresa puede determinar sus resultados comparando los ingresos obtenidos con las compensaciones y su rentabilidad en relación al capital invertido. Es posible que en estas condiciones se desarrolle una 
gestión comercial de la economía. Pero también será posible si se regula el mercado atendiendo a razones de un orden político-social, imponiendo limitaciones al acceso al mercado, premisas administrativas de precio, calidad, condición y responsabilidades del producto, obligaciones publicitarias, etc., dado que, incluso cuando se den las restricciones mencionadas, es posible que se cumpla el principio de equivalencia y dirigir la empresa de forma comercial. Tanto las ramas económicas de vinculación pública en los suministros eléctricos como la red de comunicaciones nos ofrecen suficientes ejemplos para ello. La actividad comercial o rentable encontrará, no obstante, su límite allí donde la prestación y la contraprestación ya no tengan relación alguna; con otras palabras, allí donde las necesidades y su cobertura se dirijan con arreglo a criterios extraeconómicos. En muchos casos ello estará justificado pero, al mismo tiempo, surge la pregunta de si así no se estará renunciando precipitadamente a prestaciones económicamente motivadas.

\section{COMPARACION DE LA EFICACIA}

El deber del titular de un establecimiento estatal es desarrollar económicamente las funciones públicas que las instancias políticas y administraciones legitimadas hayan determinado, cuya realización presenta varias posibilidades: si actúa a través de instituciones públicas, puede llevarlas a cabo él mismo por medio de las organizaciones locales o del Land o hacer que éstas las hagan o, en fin, encomendándoselas, bajo su supervisión, a empresas públicas. La naturaleza de las funciones determinará la vía a seguir: bien la de la planificación presupuestaria, prestaciones públicas y actividades administrativas o bien una solución empresarial que exige como condición que se pueda planificar autónomamente en el marco de unos datos estatales y que se puedan ofrecer las prestaciones con una mayor o menor adaptación al mercado.

Entre estas dos soluciones mencionadas no parece haber ninguna vía intermedia convincente. Si el Estado no quiere que el cumplimiento de sus funciones lo realicen sujetos o instituciones privadas, ni cámaras, asociaciones o sindicatos, etc., entonces él mismo debería pasar a actuar como Administración o poner en marcha una empresa pública. En la realidad, sin embargo, suelen aparecer también aquí formas mixtas, especialmente en los establecimientos públicos. Externamente se les ha concedido una independencia económico-financiera, contable, organizativa, en parte incluso jurídica, para que formalmente operen como empresas; pero en las relaciones (internas) con el titular están sometidos a menudo a unas obligacio- 
nes jurídicas presupuestarias, de caja, organizativas y de servicio tan rigurosas que terminan mereciendo la calificación de simples Administraciones subordinadas.

No obstante, no debe entenderse aquí que estoy criticando de antemano la actividad administrativa. Me expreso solamente en contra de aquellas actividades burocráticas que, en el supuesto de ser empresariales, serían a menudo más eficaces. Pero proporcionar los datos que demuestren lo que se está exponiendo no es una labor tan sencilla. En definitiva, todo quedaría reducido a una comparación de la eficacia en el cumplimiento de las tareas estatales por parte de la Administración pública o de una empresa pública, teniendo en cuenta todos los posibles efectos. Ello quiere decir que el cumplimiento alternativo de las funciones deberá ser calificado en atención a puntos de vista jurídicos, empresariales, económicos y sociales. Y centrándonos en los Studentenwerke (centros de ayuda al estudiante, ordinariamente autogestionados), se podría formular la pregunta de la manera siguiente: ¿Podrán realizar y ofrecer éstos sus prestaciones de manera más eficiente si estuviesen sometidos al Derecho administrativo, de servicios y presupuestario o si se les dejase cierta autonomía en el marco de las funciones encomendadas? Pero este análisis global de eficacia excedería de las pretensiones de este trabajo, ya que sólo puede realizarse basándose en datos empíricos, de los que no disponemos, y ello requeriría, a su vez, encontrar Studentenwerke de uno y otro tipo que estuvieren dispuestos a someterse a análisis experimentales. Así pues, me limitaré aquí, por tanto, a apuntar las ventajas económicas de una gestión empresarial.

\section{OBJETIVOS DE LA PRESTACION}

La gestión empresarial depende directamente de la formulación de los objetivos, es decir, de la especificación de aquellas situaciones que se desea alcanzar. Fijar los objetivos de una empresa es una labor mucho más sencilla que lo que pudiera ser la determinación de los objetivos públicos o, en su caso, los políticos (estatales, sociales, económicos o técnicos); especialmente debido a que la empresa es abarcable y está más próxima a sus objetivos. La posición tradicional de predominio de los juristas en las Administraciones públicas hace que la actividad se concentre en la aplicación e interpretación de las normas jurídicas y el tratamiento de las funciones públicas entendidas como actividades o como ámbitos de actividad. La transformación de los objetivos de las funciones públicas en objetivos de la Administración, concretamente en objetivos de suministros, elaboración y facilitación de prestaciones, así como de financiación, re- 
sulta — si me permiten la expresión - un lujo ciertamente prescindible para un personal administrativo que ha recibido una formación totalmente distinta. ¿Para qué se necesitan objetivos si se trabaja con unas tareas ya prescritas? Esta pregunta no puede desconcertar a los empresarios. Actuar en términos económicos supone escoger -entre el marco de posibilidades que ofrece el sistema legalla alternativa más económica o en su caso la más rentable. Pero las valoraciones de las alternativas dependen, claro está, de los objetivos que deban ser alcanzados. En los establecimientos estatales estos objetivos serán sobre todo de tipo material o de prestaciones (denominadas en general como encargos de prestaciones) que se elaboran bajo unas exigencias financieras determinadas y se plasman en objetivos financieros o formales.

Resulta verdaderamente inimaginable que fuese posible que los establecimientos estatales operasen en términos económicos sin contar para ello con un sistema de objetivos estringente e isomorfo, pues es desde éste desde el que deben ser deducidas las estrategias básicas, las diferentes funciones y las medidas concretas. Para poder calificar el grado de obtención de un resultado es indispensable que se hagan unas precisiones muy concretas. Esta concretización será el resultado de la transformación de unos objetivos a largo plazo, pasando por unos objetivos medios, en unos objetivos concretos de trabajo, de tal manera que los objetivos de prestaciones nos ofrecen así, además de su parte institucional, otra de carácter personal. Es falsa, por tanto, la observación, relativamente extendida, de que en la Administración existan planes de distribución de trabajo que especifiquen las tareas a realizar por cada uno de los funcionarios, dado que en ellos no se describen los objetivos de trabajo. Los objetivos de trabajo que se derivan del management representan una dirección de personal a través de una asignación de los objetivos. Estos tendrán carácter parcial, formulados y precisados de tal forma que puedan ser realizados. El enunciado de una prescripción del objetivo practicable podría ser la que sigue: las tareas han de ser llevadas a cabo con responsabilidad propia en un tiempo determinado y cumpliendo con un estándar determinado de su cuantía y calidad; su preparación y ejecución deberá realizarse en colaboración con un colega determinado y el resultado del trabajo deberá ser supervisado por el superior jerárquico, tanto si se trata de supuestos rutinarios como extraordinarios.

\section{PRINCIPIO DE CONGRUENCIA}

Este principio debe ser aquí entendido en el sentido de que determinados supuestos han de ser reducidos, en lo posible, a identidad o coincidencia. Atendiendo a criterios económicos quiere esto 
decir que tienen que corresponderse entre sí las funciones, los gastos y las aportaciones, o sea, que la fuerza financiera ha de garantizar la existencia material, pues ésta conlleva la capacidad financiera de la empresa o de la institución de que se trate en cada caso. El actual sistema financiero de compensación, con sus discrepancias entre el volumen de funciones y la soberanía de ingresos, así como sus financiaciones mixtas, contradice, al menos en lo que se refiere a las corporaciones territoriales, este principio, dado que la indicada congruencia financiera y de rendimientos no se da en la mayoría de Administraciones públicas.

En las empresas privadas será también más sencillo alcanzar una congruencia organizativa mayor, puesto que la distribución de las funciones, competencias y responsabilidades depende de la articulación de las distintas unidades o de una eventual participación en los resultados.

Finalmente también sería deseable que el sujeto se identificara en cierta medida con el objeto, es decir, que se buscase una especie de congruencia económico-personal, lo que se dará con mayor facilidad en empresas de tamaño reducido.

Por otra parte, la exclusión rigurosa y sistemática de funciones parciales que lleva a cabo la Administración resulta casi incompatible con una gestión comercial, infringiendo, a su vez y en parte, tanto la congruencia financiera como la organizativa y la económicopersonal antes citadas. Una empresa en la que las decisiones sobre urbanización e inversión se toman por fuerzas externas y su poder de disposición sobre los pagos y la planificación de la disponibilidad líquida se lleva a cabo por terceras instancias ajenas a las empresas es comparable a un cuerpo sin miembros. Y la gestión empresarial no es la adecuada para dirigir a un torso. El servicio de salvamento nos ofrece, ciertamente, un ejemplo clarificador. Si se entra en el tema de los titulares y de las instituciones del servicio de salvamento, aparecerán una serie de incongruencias que serán expuestas seguidamente: los órganos decisorios se ocupan de las cuestiones fundamentales y asumen la responsabilidad general, el titular de las funciones se ocupa de la ejecución del servicio, el titular de la financiación asume los costes y, finalmente, nos queda una actividad de información y representación de intereses de la que se ocupan las organizaciones de afectados. Una realización de carácter empresarial de funciones de carácter público sólo será posible si se dispone de unidades muy definidas. En parte nos encontramos ante órganos de administración propia que disponen de derechos y obligaciones propias como pueden ser, por ejemplo, universidades, municipios, seguros sociales, así como cámaras económicas. Pero de nuevo nos encontramos ante la imagen de un cuerpo mutilado por causa de la ex- 
clusión de algunas funciones parciales, aunque esenciales, o por la imposibilidad de poder disponer de fuentes propias de ingresos o por la presencia de un control ampliado e incluso de controles técnicos con directivas individuales.

¿A qué se debe que haya tantos municipios que han excluido su actividad de fomento económico de los órganos públicos encargados de la propiedad, derechos, impuestos, administración y desarrollo urbanístico, para en su lugar encomendársela a una sociedad de responsabilidad limitada de fomento económico? La respuesta es sencilla: aquí se requiere una actuación conjunta del sujeto y de los medios, decisiones rápidas, flexibilidad y una responsabilidad del resultado en general. Para evitar malentendidos quiero añadir que tampoco se puede desnaturalizar la sociedad de responsabilidad limitada de fomento económico para convertirla en un fin en sí misma. Pues ésta está sometida a las funciones que le asigne su titular y de él dependerá, en última instancia, que los objetivos asumidos obtengan resultados beneficiosos a través de las actividades comerciales. Claro que, en vez de proyectar objetivos sobre medidas y dimensiones del desarrollo industrial y tecnológico, sobre la creación de puestos de trabajo, el aumento de ingresos, reserva de suelos o sobre la adecuación al medio ambiente, el Ente matriz se limitará, por regla general, a actuaciones burocráticas imponiendo límites presupuestarios y reservándose autorizaciones y potestades de instrucción. El ejemplo resulta sintomático: ¿a quién le puede extrañar, por tanto, que a la vista de la ausencia de objetivos fijados por el titular, la dirección de la empresa municipal o del establecimiento estatal pase a articular por sí misma los objetivos públicos y, con ello, los objetivos empresariales que de ello se derivan, aun arriesgándose en esta actuación a un built-in-conflict con el Ente matriz? Este puede que llegue a tolerar estos objetivos pero, finalmente, logrará imponer por vía burocrática sus propios criterios.

\section{INCENTTVOS DE PRODUCCION}

Mediante este mecanismo de las reglas de gestión empresarial se trata de dirigir el comportamiento del personal de tal manera que intensifique su capacidad y disposición productiva con el fin de obtener un buen resultado para la empresa o para el establecimiento. Son cuatro los tipos de incentivos que se suelen distinguir, a saber: incentivos de carácter social, de carrera, monetarios e institucionales.

La denominación de incentivos sociales se refiere concretamente a las posibles recompensas para uno o varios trabajadores. Esto se puede obtener mediante una gestión adecuada a las funciones a rea- 
lizar, que domina la orientación de comportamiento y los mecanismos de imposición de su voluntad, así como la acentuación de la pertenencia al grupo a través de un fortalecimiento de la solidaridad. En los incentivos de carrera se incluyen medidas de incremento de producción que pueden preparar o dar lugar directamente a un ascenso. En el primer supuesto se trata esencialmente de personas que se encuentran todavía en formación; en el segundo, en cambio, el incentivo será el anuncio de un posible ascenso o éste mismo. Los incentivos monetarios contemplan aumentos de sueldos e ingresos que se determinan por los resultados obtenidos. Se habla finalmente de incentivos institucionales cuando a lo que se alude es a una situación privilegiada jurídica o fáctica en procesos de decisión de determinadas unidades organizativas.

Claro que, incluso en supuestos de dirección empresarial de establecimientos estatales, no podemos contar, sin más, con una flexibilidad equiparable a la de las empresas privadas; no obstante, también los establecimientos estatales disponen de ciertos incentivos monetarios e institucionales para obtener una mejor producción y éstos no se limitan al sistema de incentivos comúnmente aplicados por los órganos públicos. Hay que tener presente que al hablar aquí de una mejor producción nos estamos refiriendo a que con el mismo trabajo se obtengan mayores beneficios (adicionales) o que se obtenga un nivel de producción equivalente reduciendo, en términos generales, los medios empleados.

Pero ¿es realmente necesario que la actuación de las Administraciones públicas y de algunos establecimientos se inspire en principios de carácter disuasorio o represivo, mientras que en las empresas privadas se actúa con incentivos positivos? En el Derecho funcionarial se regula con detalle toda una gama de incentivos negativos como las posibles sanciones por una producción insuficiente, advertencias, amonestaciones, multas, recortes de salarios, responsabilidades en vía de regreso, traslados, separaciones de servicio y hasta incluso reducciones o denegaciones de la pensión. También es cierto que la Administración conoce, además, cierto tipo de elogios, ascensos y aumentos de sueldo, pero aún no se ha estudiado hasta qué punto tales medidas producen realmente y a largo plazo unos rendimientos adicionales. No obstante, queremos anotar en este lugar que el sistema de incentivos que se aplica en los establecimientos estatales con dirección empresarial es mucho más amplio que cuando éstos están dirigidos por autoridades y atendiendo a criterios domésticos, con la correspondiente aplicación de las potestades que de ello se derivan. 
DA-1989, núms. 218-219. PETER EICHHORN. La gestión empresarial aplicada a los establecimie...

\section{DETERMINACION DEL RESULTADO}

El pensamiento empresarial es, en definitiva, idéntico a un pensamiento de resultado o de éxito, referido al éxito global de toda la empresa durante el plazo completo de su existencia y, a corto plazo, en relación a los resultados de un período determinado. El éxito empresarial se determina por la diferencia entre rendimientos y gastos y, en un sentido más estricto de éxito o resultado empresarial, por la diferencia entre los ingresos y los costes de actividad. El resultado positivo se denomina ganancia o, empleando términos más restrictivos, ganancia empresarial; el resultado negativo, pérdidas o pérdidas empresariales. El éxito de un sujeto económico se traduce en la produccion de intereses del capital propio en un período determinado y, relacionando éstos, se obtiene la rentabilidad. Esta no es un objetivo a alcanzar por las Administraciones, pero sí por las empresas privadas, ya que su propia conservación depende de ello. Tratándose de una realización empresarial de funciones públicas en establecimientos estatales (o secciones de éstas) no se pretenderán alcanzar, en un principio, las máximas ganancias, teniendo que procurar garantizar, más bien y sobre todo, el mantenimiento de la capacidad de actuación de la institución. El objetivo podría ser, por tanto el siguiente: maximización de producción dándose al mismo tiempo una cobertura completa de los gastos (por ejemplo, teniendo en cuenta los descuentos oportunos y los intereses del propio capital).

Un presupuesto indispensable para poder determinar el resultado es disponer de más unidades independientes que puedan ser calculables. Esto es algo que ya se ha reconocido hace tiempo por los consorcios y las grandes empresas, creando a estos efectos centros de coste, centros de beneficios, cálculo de secciones, en parte, incluso, cálculos de puestos de trabajo. Pudiendo precisarse así los puntos de creación o disminución de resultado. Pero el resultado empresarial no se puede determinar con ingresos y gastos mediante los planes ni los presupuestos domésticos. Aparte de distribuciones y agrupaciones basadas en otro tipo de funciones (políticas, jurídicas o económicas), suele faltar, generalmente, el nexo causal entre los ingresos y los gastos, sin el cual no puede determinarse con certeza el resultado, ya que depende de la relación resultante entre prestación y contraprestación que se plasmará en los gastos y los rendimientos o en costes e ingresos.

Los establecimientos estatales que reciben un pago por sus prestaciones obran correctamente cuando determinan sus resultados comerciales sin atenerse a la contabilidad cameralística y rendición de cuentas. El resultado (anual) se señala ex-ante en el plan económico y expost en los cálculos de pérdidas o del resultado de las empresas. Así, 
por ejemplo, el plan económico de los centros de ayuda al estudiante cumple una importante función informativa y de decisión para sus Entes matrices y para los órganos de éstos. Este plan económico mencionado se compone, en definitiva, de un plan de resultados, un plan financiero y una relación de los puestos de trabajo. El primero queda estructurado según grupos de gasto y de rendimiento, establecidos de forma análoga a los cálculos de ganancias y pérdidas. El plan financiero recoge las modificaciones del patrimonio y del capital y en la relación de los puestos de trabajo aparecen los que corresponden a los empleados y a los trabajadores.

Desde una perspectiva empresarial hay que criticar, no obstante, que los planes financieros y las relaciones de los puestos de trabajo de los centros de ayuda a los estudiantes no ofrecen una muestra clara de su situación real financiera ni de sus puestos de trabajo. Y ello porque los planes financieros a menudo no incluyen las residencias, los comedores ni las cafeterías creadas y financiadas por el Bund y/o por los Länder (ya que se incluyen en el plan presupuestario individual de la Administración financiera general) y, por otro lado, tampoco recogen los puestos de trabajo de los funcionarios (que aparecen, a su vez, en el plan presupuestario estatal). Una mención de todo ello, aunque solamente fuese a título informativo, facilitaría el entendimiento y aclararía la interrelación global. Y mientras se subvencionen las inversiones y no se compensen los descuentos faltará también en el plan de resultado una categoría de gastos. Los principios del plan económico son en gran parte imperativos y se aplicarán, también, «en lo que sea posible», la legislación presupuestaria del Land, los reglamentos administrativos y órdenes de asignaciones, etc.; en concreto y especialmente, pues, prescripciones materiales y temporales. No obstante, se podrán incluir algunas facilidades en forma de anotaciones declarativas de que algunos conceptos podrán ser equiparables o transferibles.

\section{SUBVENCIONES GLOBALES}

Queda, por tanto, en manos de la ley, del reglamento y de aquel a quien corresponde realizar las asignaciones financieras y presupuestarias la determinación de si y hasta qué punto un centro de ayuda al estudiante podrá ser gestionado de forma empresarial o si ha de atenerse al rigor de prescripciones, autorizaciones, instrucciones y controles. Por mi parte creo que una mayor libertad creativa conllevaría una mejor gestión empresarial de los centros de ayuda al estudiante. Lo cual supone una fijación previa de los objetivos y, con sujeción a ellos, la transferencia de ámbitos libres de decisión y actuación. Pero hay que aceptar, no obstante, que este margen al que se alude tiene 
que ser más reducido cuando se trate de sectores subvencionados que en los ámbitos de las llamadas competencias propias; aunque debería desaparecer globalmente en favor de una mayor flexibilidad. En el seno interno de los centros de ayuda al estudiante podrá llevarse a cabo por parte del Consejo de Administración y de la Gerencia la diferenciación presupuestaria necesaria para comedores, residencias, creación de viviendas, programas de tutores, guarderías, asesorías psicoterapéuticas y administración.

Traducción: Julia NIETo KoENIG 
DA-1989, núms. 218-219. PETER EICHHORN. La gestión empresarial aplicada a los establecimie...

DA-1989, núms. 218-219. PETER EICHHORN. La gestión empresarial aplicada a los establecimie... 